\title{
Novel agent induction therapy alone or followed by autologous stem cell transplantation in younger patients with multiple myeloma: A single-center retrospective study of 114 cases
}

\author{
YAN WANG, PENGPENG XU, YUBAO CHEN, QINGYE FAN, \\ JUNMIN LI, WEILI ZHAO, JIANQING MI and HUA YAN
}

Department of Hematology, Ruijin Hospital, Shanghai Jiao-Tong University School of Medicine, Shanghai 200025, P.R. China

Received February 16, 2015; Accepted October 5, 2015

DOI: $10.3892 / \mathrm{mco} .2015 .658$

\begin{abstract}
To define the role of autologous stem cell transplantation (ASCT) in newly diagnosed multiple myeloma (MM) in the era of novel agents, we analyzed follow-up data of patients treated by these agents alone or followed by ASCT. From January, 2008 to December, 2012, 136 patients with de novo MM, aged $<65$ years, completed bortezomib- or thalidomide-based induction therapy and 114 patients achieved at least a partial response (PR). A total of 42 patients underwent ASCT. After a median follow-up of 39 months (range, 5-74 months), the median progression-free survival (PFS) was 23 months in the non-ASCT group vs. 42 months in the ASCT group $(\mathrm{P}=0.001)$, and the 5-year overall survival (OS) rate was 58.9 vs. $81.2 \%$, respectively $(\mathrm{P}=0.03)$. The multivariate analysis revealed that complete response (CR) and maintenance therapy (MT) were independent factors of improved OS in both groups. Moreover, a subgroup analysis was performed according to the response status to evaluate the role of ASCT and MT. In the CR subgroup, neither ASCT nor MT exerted a significant effect on PFS or OS. In the very good PR subgroup, ASCT after MT (ASCT/MT) significantly improved PFS, but not OS. In patients exhibiting PR, ASCT/MT significantly prolonged PFS and OS. Therefore, ASCT in the era of novel agents maintains an important role in younger MM patients, particularly those achieving a PR after induction therapy. Furthermore, MT is a key factor associated with long-term survival in all MM patients.
\end{abstract}

\section{Introduction}

Multiple myeloma (MM) is the main type of hematological malignancy originating from plasma cells. MM is currently

Correspondence to: Professor Hua Yan, Department of Hematology, Ruijin Hospital, Shanghai Jiao-Tong University School of Medicine, 197 Ruijin Er Road, Shanghai 200025, P.R. China E-mail: yanhua_candy@163.com

Key words: multiple myeloma, autologous hematopoietic stem cell transplantation, maintenance treatment, progression-free survival, overall survival an incurable disease. With conventional chemotherapeutic regimens, such as melphalan and prednisone (MP), vincristine plus adriamycin and dexamethasone (VAD) and high-dose dexamethasone, the overall response rate (RR) of MM patients is $60 \%$, with only $<5 \%$ achieving a complete response (CR). The median survival is 2-3 years (1). High-dose chemotherapy followed by autologous stem cell transplantation (ASCT) may achieve a higher RR and longer progression-free survival (PFS) and overall survival (OS) (2-4). Over the last decade, the introduction of the proteasome inhibitor bortezomib and the immunomodulatory agents thalidomide and lenalidomide, has revolutionised MM treatment $(5,6)$. The high $\mathrm{RR}$ and $\mathrm{CR}$ rate achieved by these novel agents have also raised the question whether ASCT should still be considered as first-line therapy in MM. Several phase 3 trials comparing chemotherapy with first-line ASCT have reported an improved PFS, but no difference in OS $(7,8)$, while others support ASCT as part of the treatment strategy $(9,10)$. It is clear that ASCT enhances the response, even after the most active first-line regimens, including bortezomib plus thalidomide and dexamethasone (VTD) and lenalidomide plus bortezomib, pegylated liposomal doxorubicin and dexametasone (RVDD) $(11,12)$. ASCT is currently considered the standard of care in younger MM patients according to the current National Comprehensive Cancer Network guidelines (13).

However, in China, the majority of the patients may be less likely to undergo transplantation due to multiple reasons, and data on which group of patients may benefit more from ASCT are currently limited. In this study, we performed a retrospective analysis of $114 \mathrm{MM}$ patients, aged $<65$ years, who were treated with or without ASCT following novel agent-containing induction therapy in our hospital.

\section{Patients and methods}

Patients. From January, 2008 to December, 2012, 136 patients with de novo MM, aged $<65$ years, received bortezomib- or thalidomide-containing induction therapy at the Department of Hematology of Ruijin Hospital (Shanghai, China). Following induction therapy, 114 patients who achieved at least a PR and had no severe comorbidities, were eligible for ASCT. Among these, 42 patients received ASCT within 1 year of diagnosis 
Table I. Baseline characteristics of the 114 multiple myeloma patients.

\begin{tabular}{|c|c|c|}
\hline Characteristics & Non-ASCT $(n=72)$ & $\operatorname{ASCT}(n=42)$ \\
\hline \multicolumn{3}{|l|}{ Age, years } \\
\hline Median (range) & $57(29-65)$ & $53(41-65)$ \\
\hline Gender, male/female & $43 / 29$ & $30 / 12$ \\
\hline \multicolumn{3}{|l|}{ M component, n (\%) } \\
\hline $\operatorname{IgG}$ & $41(57.0)$ & $25(59.5)$ \\
\hline $\operatorname{IgA}$ & $19(26.4)$ & $6(14.3)$ \\
\hline $\operatorname{IgD}$ & $2(2.7)$ & $5(11.9)$ \\
\hline Light chain & $10(13.9)$ & $6(14.3)$ \\
\hline \multicolumn{3}{|l|}{ DS stage, n (\%) } \\
\hline I & 0 & 0 \\
\hline II & $15(20.8)$ & $14(33.3)$ \\
\hline III & $57(79.2)$ & $28(66.7)$ \\
\hline \multicolumn{3}{|l|}{ ISS stage, n (\%) } \\
\hline I & $1(1.4)$ & $1(2.4)$ \\
\hline II & $61(84.7)$ & $32(76.2)$ \\
\hline III & $10(13.9)$ & $9(21.4)$ \\
\hline Number of induction cycles, median (range) & $5(2-10)$ & $4(3-8)$ \\
\hline \multicolumn{3}{|l|}{ Best response after induction therapy, n (\%) } \\
\hline $\mathrm{CR}$ & $19(26.4)$ & $13(31.0)$ \\
\hline VGPR & $22(30.6)$ & $14(33.3)$ \\
\hline PR & $31(43.0)$ & $15(35.7)$ \\
\hline \multicolumn{3}{|l|}{ Induction regimen, $\mathrm{n}(\%)$} \\
\hline Bortezomib & $55(76.4)$ & $25(59.5)$ \\
\hline Thalidomide & $17(23.6)$ & $17(40.5)$ \\
\hline \multicolumn{3}{|l|}{ Maintenance therapy, n (\%) } \\
\hline Yes & $40(55.6)$ & $25(59.5)$ \\
\hline No & $32(44.4)$ & $17(40.5)$ \\
\hline
\end{tabular}

ASCT, autologous hematopoietic stem cell transplantation; DS, Durie-Salmon staging system; ISS, International Staging System; CR, complete response; PR, partial response; VGPR, very good PR.

(ASCT group). The remaining 72 patients declined ASCT for personal reasons (non-ASCT group). This study was approved by the Ethics Committee of Ruijin Hospital and conformed to the principles of the Declaration of Helsinki. Written informed consent was obtained from all the patients.

Evaluation. MM was diagnosed according to the uniform response criteria of the International Myeloma Working Group (IMWG) (14). All 114 patients had symptomatic MM, with measurable disease. Non-secretory MM cases were excluded. The response was classified as complete response (CR), very good partial response (VGPR), partial response (PR) and progressive disease (PD), according to the IMWG criteria.

Treatment regimens. All the patients were induced with chemotherapeutic regimens, including bortezomib plus adriamycin and dexamethasone (PAD), or bortezomib plus cyclophosphamide and dexamethasone (PCD), or VAD and thalidomide (VADT). Bortezomib was administered at a dose of $1.3 \mathrm{mg} / \mathrm{m}^{2}$ i.v. twice/week for 2 weeks in a 21- or 28-day cycle. Thalidomide was administered at a dose of $100 \mathrm{mg}$ daily. In the ASCT group, all the patients received peripheral blood progenitor cell mobilization with cyclophosphamide $\left(4 \mathrm{~g} / \mathrm{m}^{2}\right)$ and conditioning therapy with high-dose melphalan $\left(100-200 \mathrm{mg} / \mathrm{m}^{2}\right)$. The main maintenance therapy (MT) regimen was thalidomide (50-150 mg/day), which, if tolerated, continued until disease progression.

Statistical analysis. PFS was calculated from the date of the initiation of induction therapy to the date of disease progression, relapse, or death. OS was measured from the date of diagnosis to the date of death; data on survivors were censored at the last follow-up. All analyses were performed with SPSS 19.0 statistical software (IBM SPSS, Armonk, NY, USA). Survival outcomes were analyzed with the Kaplan-Meier method and compared with the log-rank test. The Cox proportional hazards model was used for multivariate analysis, and all the variables achieving $\mathrm{P}<0.25$ in the univariate ananlysis were considered.

\section{Results}

Patient characteristics. The study population comprised 114 patients, of whom $73(64 \%)$ were male. The median age 
Table II. Univariate analysis of the prognostic factors for survival in the non-ASCT group.

\begin{tabular}{|c|c|c|c|c|c|}
\hline Factors & $\mathrm{n}$ & PFS (months) & P-value & OS (months) & P-value \\
\hline DS stage & & & 0.600 & & 0.357 \\
\hline II & 15 & $24 \pm 3.618$ & & NR & \\
\hline III & 57 & $23 \pm 2.589$ & & $65 \pm 15.156$ & \\
\hline ISS stage & & & 0.004 & & 0.171 \\
\hline $\mathrm{I}+\mathrm{II}$ & 62 & $26 \pm 2.828$ & & $65 \pm 8.145$ & \\
\hline III & 10 & $15 \pm 2.324$ & & $42 \pm 19.322$ & \\
\hline Best response after induction therapy & & & 0.010 & & 0.007 \\
\hline $\mathrm{CR}$ & 19 & $38 \pm 9.413$ & & NR & \\
\hline VGPR & 22 & $23 \pm 4.462$ & & $65 \pm 4.605$ & \\
\hline PR & 31 & $18 \pm 2.226$ & & $42 \pm 10.882$ & \\
\hline Induction regimen & & & 0.982 & & 0.765 \\
\hline Bortezomib & 54 & $23 \pm 2.902$ & & NR & \\
\hline Thalidomide & 18 & $25 \pm 3.135$ & & $55 \pm 9.832$ & \\
\hline Maintenance therapy & & & 0.015 & & 0.004 \\
\hline Yes & 40 & $27 \pm 2.726$ & & NR & \\
\hline No & 32 & $17 \pm 2.62$ & & $36 \pm 6.924$ & \\
\hline
\end{tabular}

The values are presented as mean \pm standard deviation. ASCT, autologous hematopoietic stem cell transplantation; DS, Durie-Salmon staging system; ISS, International Staging System; CR, complete response; PR, partial response; VGPR, very good PR; PFS, progression-free survival; OS, overall survival; NR, not reached.
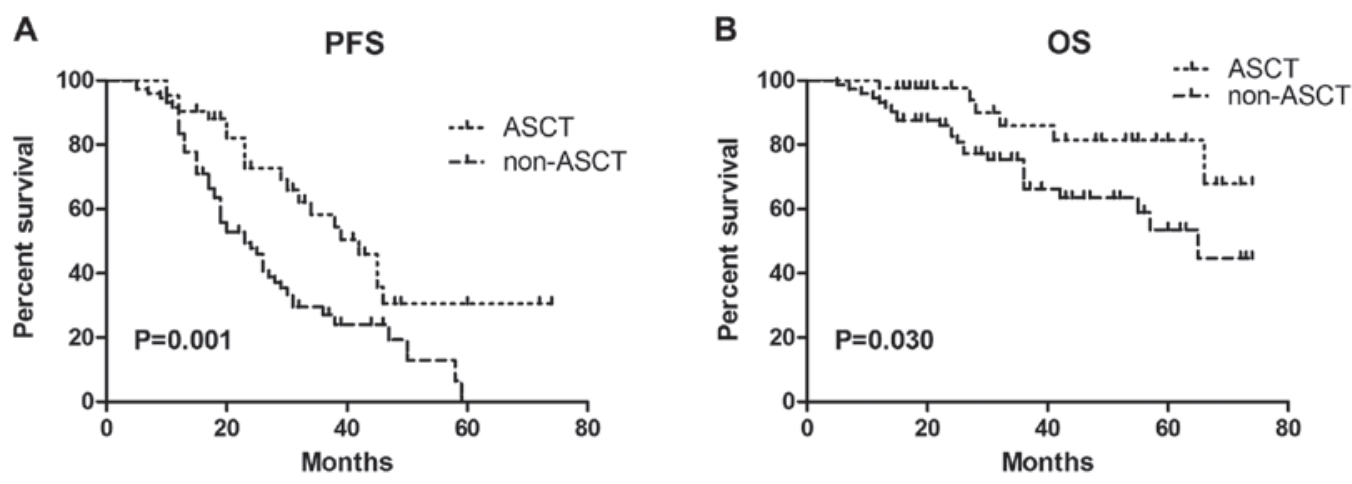

Figure 1. (A) PFS and (B) overall OS were superior in the ASCT group compared with those in the non-ASCT group. PFS, progression-free survival; OS, overall survival; ASCT, autologous hematopoietic stem cell transplantation.

of the patients was 56 years (range, 26-64 years) and $85(75 \%)$ patients had Durie-Salmon stage III disease at diagnosis. A total of 80 patients received bortezomib-containing induction therapy, whereas the remaining patients received thalidomide-containing therapy. Following induction therapy, all the patients underwent response evaluation: A total of 32 patients had achieved CR, 36 patients had VGPR and 46 patients had PR. Of the 114 patients, 42 received high-dose chemotherapy followed by ASCT (ASCT group), whereas the remaining 72 patients did not receive ASCT (non-ASCT group). The baseline characteristics of the ASCT and non-ASCT groups are listed in Table I. No significant difference in clinical characteristics was observed between the two groups.

Disease outcome. The median follow-up time of the 114 patients was 39 months (range, 5-74 months). At the time of the last follow-up (March 31st, 2014), 52 of the 72 (72.2\%) patients in the non-ASCT group had progressed or relapsed, whereas 24 patients $(33.3 \%)$ had succumbed to the disease. In the ASCT group, 20 of the $42(47.6 \%)$ patients had progressed or relapsed and 6 patients $(14.3 \%)$ had succumbed to the disease. The median PFS in the non-ASCT and ASCT groups was 23 and 42 months, respectively $(\mathrm{P}=0.001$, Fig. $1 \mathrm{~A})$. The median OS was not reached in neither of the groups. The 5-year OS rate was 58.9 and $81.2 \%$ in the non-ASCT and ASCT groups, respectively ( $\mathrm{P}=0.03$, Fig. 1B).

Prognostic indicators. The univariate analysis in the non-ASCT group (Table II) revealed that the prognostic factors associated with prolonged PFS and OS included CR $(\mathrm{P}=0.01$ and 0.007 , respectively) and MT $(\mathrm{P}=0.015$ and 0.004 , respectively). Lower International Staging System (ISS) stage 
Table III. Univariate analysis of the prognostic factors for survival in the ASCT group.

\begin{tabular}{|c|c|c|c|c|c|}
\hline Factors & $\mathrm{n}$ & PFS (months) ${ }^{\mathrm{a}}$ & P-value & 3-year OS (\%) & P-value \\
\hline DS stage & & & 0.903 & & 0.407 \\
\hline II & 14 & $42 \pm 7.574$ & & 81.3 & \\
\hline III & 28 & $39 \pm 7.907$ & & 88.5 & \\
\hline ISS stage & & & 0.781 & & 0.625 \\
\hline $\mathrm{I}+\mathrm{II}$ & 33 & $42 \pm 6.674$ & & 86.7 & \\
\hline III & 9 & $38 \pm 10.474$ & & 83.3 & \\
\hline Response after ASCT & & & 0.020 & & 0.067 \\
\hline CR & 29 & $45 \pm 2.553$ & & 94.7 & \\
\hline VGPR & 10 & $30 \pm 4.714$ & & 62.5 & \\
\hline PR & 3 & $17 \pm 5.715$ & & 66.7 & \\
\hline Maintenance therapy & & & 0.408 & & 0.038 \\
\hline Yes & 25 & $42 \pm 4.812$ & & 92.9 & \\
\hline No & 17 & $34 \pm 5.837$ & & 77.0 & \\
\hline
\end{tabular}

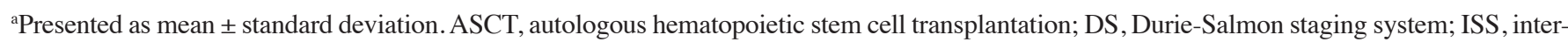
national staging system; CR, complete response; PR, partial response; VGPR, very good PR; PFS, progression-free survival; OS, overall survival.
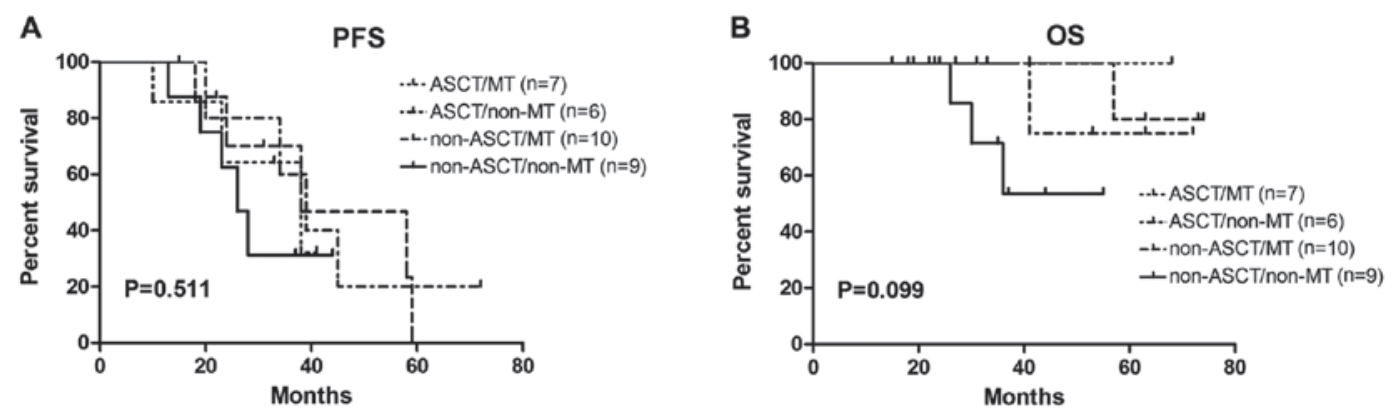

Figure 2. ASCT and MT did not significantly affect the outcome of the patients achieving a complete response prior to ASCT. (A) The median PFS \pm standard deviation of the four arms (ASCT/MT, ASCT/non-MT, non-ASCT/MT, non-ASCT/non-MT) was 38 $\pm 11.688,39 \pm 6.736,58 \pm 15.88$ and $26 \pm 2.947$, respectively. (B) The 3-year OS rate of the four arms (ASCT/MT, ASCT/non-MT, non-ASCT/MT, non-ASCT/non-MT) was 100, 80, 80 and $53.6 \%$, respectively. PFS, progression-free survival; OS, overall survival; ASCT, autologous hematopoietic stem cell transplantation; MT, maintenance therapy.

(I+II) was associated with superior PFS $(\mathrm{P}=0.004)$, but not OS $(\mathrm{P}=0.171)$.

The univariate analysis in the ASCT group (Table III) revealed that the prognostic factors of a favorable outcome included $C R$ post-ASCT, which prolonged $\mathrm{PFS}(\mathrm{P}=0.02)$, but not OS $(\mathrm{P}=0.067)$. MT significantly prolonged OS $(\mathrm{P}=0.038)$.

The multivariate analysis revealed that, for all the patients, CR post-induction therapy, ASCT and MT were prognostic factors of improved PFS and OS, whereas ISS stage affected PFS, but not OS (Table IV).

Effect of ASCT and MT on subgroups. Furthermore, we performed a subgroup analysis base on the response evaluation. Following induction therapy, the patients were divided into three subgroups, namely the CR, VGPR and PR subgroups. For each subgroup, the effect of ASCT and MT on PFS and OS was analyzed. According to the two factors (ASCT and MT), four arms were formed as follows: ASCT/MT, ASCT/non-MT, non-ASCT/MT, and non-ASCT/non-MT. In the CR subgroup (Fig. 2), no significant differences in PFS and OS were observed
Table IV. Multivariate analysis of prognostic factors for survival in all multiple myeloma patients.

\begin{tabular}{lccc}
\hline Variables & P-value & HR & $95 \%$ CI \\
\hline PFS & & & \\
$\quad$ ISS stage III vs. I-II & 0.009 & 2.202 & $1.223-3.965$ \\
No CR vs. CR & 0.004 & 2.284 & $1.301-4.011$ \\
No ASCT vs. ASCT & 0.000 & 2.871 & $1.659-4.966$ \\
No MT vs. MT & 0.002 & 2.170 & $1.336-3.525$ \\
OS & & & \\
ISS stage III vs. I-II & 0.720 & 1.184 & $0.471-2.978$ \\
No CR vs. CR & 0.041 & 2.743 & $1.040-7.233$ \\
No ASCT vs. ASCT & 0.023 & 2.912 & $1.161-7.305$ \\
No MT vs. MT & 0.001 & 3.917 & $1.795-8.549$ \\
\hline
\end{tabular}

PFS, progression-free survival; OS, overall survival; ISS, International Staging System; CR, complete response; ASCT, autologous hematopoietic stem cell transplantation; MT, maintenance therapy. $\mathrm{HR}$, hazard ratio; CI, confidence interval. 
A

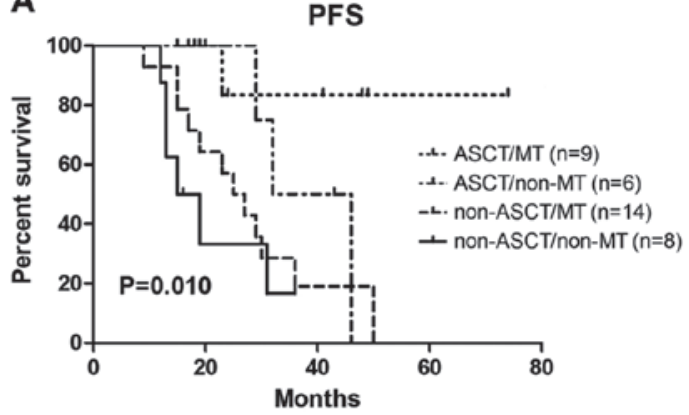

B

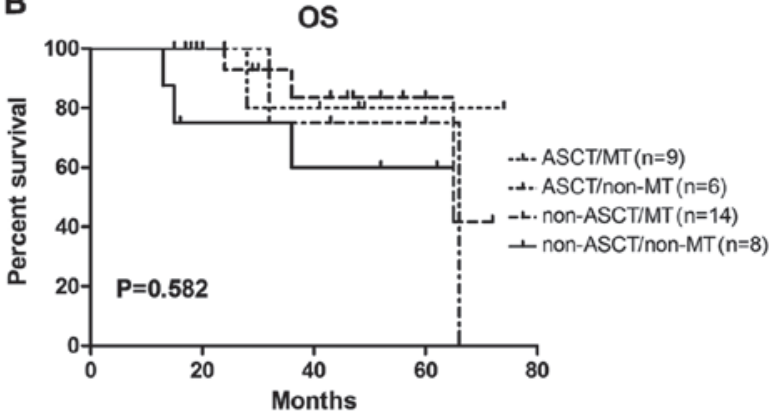

Figure 3. ASCT and MT affected PFS but not OS in the patients achieving a very good partial response prior to ASCT. (A) The median PFS \pm standard deviation of the four arms (ASCT/MT, ASCT/non-MT, non-ASCT/MT, non-ASCT/non-MT) was NR (not reached), 32 $\pm 5.667,25 \pm 3.742,15 \pm 3.637$, respectively. (B) The 3-year OS rate of the four arms was 80, 75, 83 and 60\%, respectively. PFS, progression-free survival; OS, overall survival; ASCT, autologous hematopoietic stem cell transplantation; MT, maintenance therapy.

A

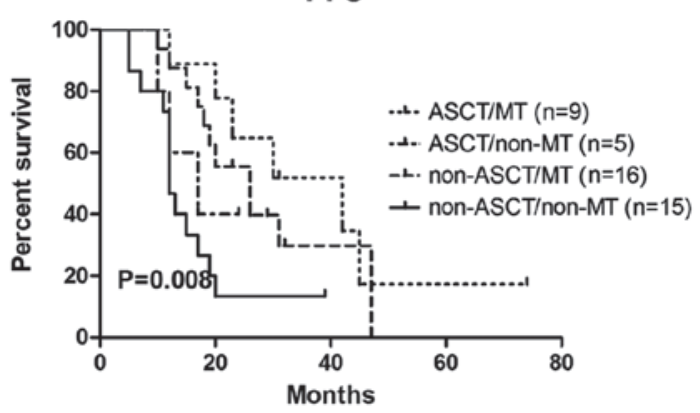

B

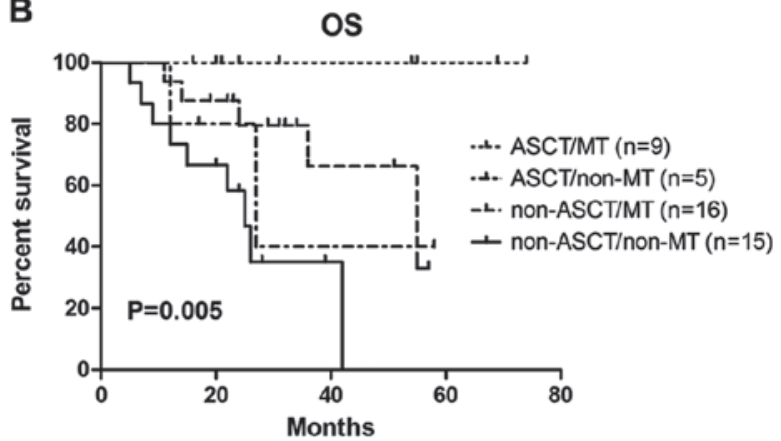

Figure 4. ASCT and MT affected both PFS and OS in the patients achieving a partial response prior to ASCT. (A) The median PFS \pm standard deviation of the four arms (ASCT/MT, ASCT/non-MT, non-ASCT/MT, non-ASCT/non-MT) was 42 $\pm 11.52,17 \pm 5.477,26 \pm 4.942$ and 12 \pm 7.73 , respectively. (B) The 3-year OS rate of the four arms (ASCT/MT, ASCT/non-MT, non-ASCT/MT, non-ASCT/non-MT) (ASCT/MT, ASCT/non-MT, non-ASCT/MT, non-ASCT/non-MT) was 100, 40, 66 and 35\%, respectively. PFS, progression-free survival; OS, overall survival; ASCT, autologous hematopoietic stem cell transplantation; MT, maintenance therapy.

among the four arms $(\mathrm{P}=0.099$ and $\mathrm{P}=0.511$, respectively); patients without ASCT but with MT still reached the median PFS of $58 \pm 15.88$ months, with a 3 -year OS rate of $80 \%$. In the VGPR subgroup (Fig. 3), PFS prolongation was achieved by the patients undergoing ASCT following MT $(\mathrm{P}=0.012)$, but there was no statistical difference in $\mathrm{OS}(\mathrm{P}=0.582)$ among the four arms. In the PR subgroup (Fig. 4), the greatest benefit from ASCT and MT was observed. There were statistically significant differences in PFS $(\mathrm{P}=0.008)$ and $\mathrm{OS}(\mathrm{P}=0.005)$ among the four arms.

\section{Discussion}

Multiple myeloma is currently considered incurable. The aim of the treatment of this disease is to prolong PFS, and eventually OS. The majority of the available studies indicate that achievement of CR was associated with prolonged PFS and OS $(6,15)$. With conventional chemotherapy, the CR rate is currently $5-8 \%$. High-dose therapy followed by ASCT increases the response rate and improves response to treatment, achieving a $\mathrm{CR}$ rate of $22-44 \%(16,17)$. More recently, novel agent-containing induction therapy has achieved a high response rate and CR rate. In the phase 3 VISTA study of bortezomib plus MP (VMP) vs. MP alone, the CR rate was $28 \%$ in the VMP group (18). In other bortezomib-containing induction regimens, the CR rate reached $22-47 \%$, with a 1 -year PFS of $83-100 \%$ (5). The improved outcome of the novel agents may challenge the role of ASCT in the treatment of MM.

In this retrospective study, CR rate reached $28 \%(32 / 114)$ with the novel-agent induction therapy. Further benefits were obtained in the ASCT group, with the CR rate increasing from $31 \%$ (pre-ASCT) to $69 \%$ (post-ASCT). The survival analysis also revealed that PFS and OS were significantly prolonged in the ASCT group, supporting the beneficial role of ASCT in younger MM patients in the era of novel agents $(19,20)$. However, different results were reported by other studies. Boccadoro et al (7) compared MP/lenalidomide (MPR) vs. ASCT plus lenalidomide maintenance or no maintenance and indicated that ASCT improved PFS, while the effect on OS was insignificant, suggesting that lenalidomide maintenance may balance the OS in the two groups. The improved OS in our study may be due to a higher number of cases achieving CR in the ASCT group ( $\mathrm{n}=29$ case, 69\%).

Thalidomide, commonly used as MT and sequential therapy, was shown to improve PFS and OS $(21,22)$. In our study, the multivariate analysis revealed that MT was an independent factor of improved PFS and OS in the non-ASCT as well as the ASCT group. However, a study conducted by Barlogie et al (23) reported a beneficial effect on PFS, but not OS and suggested that the similar OS between the two groups is partially due to the shorter survival following relapse in the thalidomide maintenance group. In addition, Attal et al $(22,24)$ 
reported an OS advantage from thalidomide maintenance with a follow-up of 39 months, while the OS advantage was lost when the follow-up was prolonged to 5.7 years. In our study, the median OS time has not yet been reached, and elucidating the survival advantages of MT requires a longer follow-up and further investigation.

CR achievement was another independent factor in our study, which was consistent with previous studies $(4,6)$. Attaining a CR has been the major objective in the management of younger patients. However, the data on whether patients who achieved CR following induction therapy may further benefit from ASCT are currently limited. We performed a subgroup analysis according to the response status following induction therapy. Considering the important role of MT on survival, we analyzed ASCT as well as MT. Interestingly, in the subgroup of CR, with or without ASCT/MT, no statistically significant difference in PFS or OS was observed. It was previously demonstrated that stringent complete response (sCR) was an attainable goal following ASCT, which significantly improved survival outcome compared with conventional CR (25). As shown in our study, a better OS (5-year OS rate of 100\%) may be achieved in the ASCT/MT arm, which may be due to more sCRs obtained, although the difference was not statistically significant. Our findings support the role of ASCT and MT in deepening the CR.

In the VGPR subgroup, ASCT and MT prolonged the PFS $(\mathrm{P}=0.010)$, but without an $\mathrm{OS}$ benefit $(\mathrm{P}=0.582)$. However, through constructing Kaplan-Meier curves, modest differences were observed among arms. Since the median OS time has not been reached, we hypothesized that different findings may emerged after long-term follow-up. In the PR subset, the patients undoubtedly benefited the most from ASCT and MT in terms of survival $(\mathrm{P}<0.01)$. The median PFS of the ASCT/MT arm was 42 months, with a 3 -year OS rate of $100 \%$, whereas in the non-ASCT/non-MT arm, the median PFS was only 12 months, with a 3 -year OS rate of $35 \%$. A poor PFS (17 months) and a 3 -year OS rate of $40 \%$ was noted in the ASCT/no-MT arm. In this arm, the sample was too small $(n=5)$ and 3 patients remained in PR following ASCT. Similar to other reports, any response less than VGPR following ASCT was always associated with a poor outcome $(26,27)$.

In summary, novel-agent induction therapy resulted in a higher response rate and response quality. ASCT further increased the CR rate and improved PFS and OS, particularly in patients achieving a PR following induction therapy. In the patients who achieved $\mathrm{CR}$ following induction therapy, the role of ASCT requires further investigation. As an independent prognostic factor of better PFS and OS, MT has become an important part of the treatment strategy in MM.

\section{Acknowledgements}

This study was supported by the National Natural Science Foundation of China (grant nos. 81302038 and 81201863).

\section{References}

1. Collaborative Group: Combination chemotherapy versus melphalan plus prednisone as treatment for multiple myeloma: An overview of 6,633 patients from 27 randomized trials Myeloma Trialists' Collaborative Group. J Clin Oncol 16: 3832-3842, 1998.
2. Harousseau JL and Attal M: The role of autologous hematopoietic stem cell transplantation in multiple myeloma. Semin Hematol 34 (1 Suppl 1): 61-66, 1997.

3. Blade J, Rosinol L, Cibera MT, Rovira M and Carreras E: Hematopoietic stem cell transplantation for multiple myeloma beyond 2010. Blood 115: 3655-3663, 2010.

4. Van de Velde HJ, Liu X, Chen G, Cakana A, Deraedt W and Bayssas M: Complete response correlates with long-term survival and progression-free survival in high-dose therapy in multiple myeloma. Haematologica 92: 1399-1406, 2007.

5. Kumar S, Flinn I, Richardson PG, Hari P, Callander N, Noga SJ, Stewart AK, Turturro F, Rifkin R, Wolf J, et al: Randomized, multicenter, phase 2 study (EVOLUTION) of combinations of bortezomib, dexamethasone, cyclophosphamide and lenalidomide in previously untreated multiple myeloma. Blood 119: 4375-4382, 2012.

6. Chanan-Kahn AA and Giralt S: Importance of achieving complete response in multiple myeloma and the impact of novel agents. J Clin Oncol 28: 2612-2624, 2010.

7. Boccadoro M, Cavallo F, Nagler A, Ben Yehuda D, Omedè P, Cavalli M, Levi A, Crippa C, Siniscalchi A, Brasca P, et al: Melphalan/prednisone/lenalidomide (MPR) versus high-dose melphalan and autologous transplantation (MEL200) in newly diagnosed multiple myeloma (MM) patients: a phase III trial. J Clin Oncol 29 (Supp 1): 8020-8027, 2011.

8. Gay F, Hajek R, Diramondo F, et al: Cyclophosphamide lenalidomide-dexamethasone vs. autologous transplant in newly diagnosed myeloma: a phase 3 trial. Clin Lymphoma Myeloma Leuk 13 (Suppl 1): S40, 2013

9. Fermand JP, Katsahian S, Divine M, et al: High-dose therapy and autologous blood stem-cell transplantation compared with conventional treatment in myeloma patients aged 55 to 65 years: long-term results of a randomized control trial from the Group Myelome-Autogreffe. J Clin Oncol 23: 9227-9233, 2005.

10. Hahn T, Wingard JR, Anderson KC, et al: The role of cytotoxic therapy with hematopoietic stem cell transplantation in the therapy of multiple myeloma: an evidence-based review. Biol Blood Marrow Transplant 9: 4-37, 2003.

11. Moreau P, Avet-Loiseau H, Facon T, Attal M, Tiab M, Hulin C, Doyen C, Garderet L, Randriamalala E, Araujo C, et al: Bortezomib plus dexamethasone versus reduced-dose bortezomib, thalidomide plus dexamethasone as induction treatment before autologous stem cell transplantation in newly diagnosed multiple myeloma. Blood 118: 5752-5758, 2011.

12. Jakubowiak AJ, Griffith KA, Reece DE, Hofmeister CC, Lonial S, Zimmerman TM, Campagnaro EL, Schlossman RL, Laubach JP, Raje NS, et al: Lenalidomide, bortezomib, pegylated liposomal doxorubicin and dexamethasone in newly diagnosed multiple myeloma: a phase 1/2 Multiple Myeloma Research Consortium trial. Blood 118: 535-543, 2011.

13. Kenneth C Anderson, Melissa Alsina, William Bensinger, et al: Multiple Myeloma, Version 1.2013 J Natl Compr Canc Netw 11: 11-17, 2013.

14. Durie BG, Harousseau JL, Miguel JS, et al: International uniform response criteria for multiple myeloma. Leukemia 20: 1467-1473, 2006.

15. Haroussear JL, Attal M and Avet-Loiseau H: The role of complete response in multiple myeloma. Blood 114: 3139-3146, 2009.

16. Attal M, Harousseau JL, Stoppa AM, Sotto JJ, Fuzibet JG, Rossi JF, Casassus P, Maisonneuve H, Facon T, Ifrah N, et al: A prospective, randomized trial of autologous bone marrow transplantation and chemotherapy in multiple myeloma. Intergroupe Français du Myélome. N Eng1 J Med 335: 91-97, 1996.

17. Child JA, Morgan GJ, Davies FE, Owen RG, Bell SE, Hawkins K, Brown J, Drayson MT and Selby PJ; Medical Research Council Adult Leukaemia Working Party: High-dose chemotherapy with hematopoietic stem-cell rescue for multiple myeloma. N Engl J Med 348: 1875-1883, 2003.

18. Harousseau JL, Palumbo A, Richardson PG, Schlag R, Dimopoulos MA, Shpilberg O, Kropff M, Kentos A, Cavo M, Golenkov A, et al: Superior outcomes associated with complete response in newly diagnosed multiple myeloma patients treated with nonintensive therapy: analysis of the phase 3 VISTA study of bortezomib plus melphalan-prednisone versus melphalan-prednisone. Blood 116: 3743-3750, 2010.

19. Reeder CB, Reece DE, Kukreti V, Chen C, Trudel S, Hentz J, Noble B, Pirooz NA, Spong JE, Piza JG, et al: Cyclophosphamide, bortezomib and dexamethasone induction for newly diagnosed multiple myeloma: high response rates in a phase II clinical trial. Leukemia 23: 1337-1341, 2009. 
20. Kumar L, Iqbal N, Mookerjee A, Verma RK, Sharma OD, Batra A, Pramanik R and Gupta R: Complete response after autologous stem cell transplant in multiple myeloma. Cancer Med 3: 939-946, 2014.

21. Spencer A, Prince HM, Roberts AW, Prosser IW, Bradstock KF Coyle L, Gill DS, Horvath N, Reynolds J and Kennedy N: Consolidation therapy with low-dose thalidomide and prednisolone prolongs the survival of multiple myeloma patients undergoing a single autologous stem-cell transplantation procedure. J Clin Oncol 27: 1788-1793, 2009.

22. Attal M, Harousseau JL, Leyvraz S, Doyen C, Hulin C, Benboubker L, Yakoub Agha I, Bourhis JH, Garderet L, Pegourie B, et al; Inter-Groupe Francophone du Myélome (IFM).: Maintenance therapy with thalidomide improves survival in patients with multiple myeloma. Blood 108: 3289-3294, 2006.

23. Barlogie B, Tricot G, Anaissie E, Shaughnessy J, Rasmussen E, van Rhee F, Fassas A, Zangari M, Hollmig K, Pineda-Roman M, et al: Thalidomide and hematopoietic cell transplantation for multiple myeloma. N Engl J Med 354: 1021-1030, 2006.
24. Attal M and Roussel M: Maintenance therapy for myeloma. How much, how long and at what cost? Am Soc Clin Oncol Educ Book 32: 515-522, 2012

25. Kapoor P, Kumar SK, Dispenzieri A, et al: Importance of achieving stringent complete response after autologous stem-cell transplantation in multiple myeloma. J Clin Oncol 31: 4529-4535, 2013.

26. Bergantim R, Trigo F and Guimaraes JE: Impact of tandem autologous stem cell transplantation and response to transplant in the outcome of multiple myeloma. Exp Hematol Oncol 1: 35-38, 2012.

27. Martinez-Lopez J, Blade J, Mateos MV, et al: Long-term prognostic significance of response in multiple myeloma after stem cell transplantation. Blood 118: 529-534, 2011. 